\title{
1 barCoder: a tool to generate unique, orthogonal genetic tags for qPCR detection
}

2 Casey B. Bernhards ${ }^{1,2^{*}}$, Matthew W. Lux ${ }^{1 *}$, Sarah E. Katoski ${ }^{1}$, Tyler D. P. Goralski ${ }^{1}$, Alvin T.

3 Liem $^{1,3}$, Henry S. Gibbons ${ }^{1 \dagger}$

$4 \quad *$ These authors contributed equally to this work

$5 \quad$ Corresponding author

$6 \quad{ }^{1}$ U.S. Army Combat Capabilities Development Command Chemical Biological Center, Aberdeen

7 Proving Ground, MD 21010, USA

$8 \quad{ }^{2}$ Excet, Inc., Springfield, VA 22150, USA

$9 \quad{ }^{3}$ DCS Corporation, Abingdon, MD 21009, USA

10

11

12

13

14

15

16 


\section{Abstract:}

25 Background: Tracking dispersal of microbial populations in the environment requires specific

26 detection methods that discriminate between the target strain and all potential natural and

27 artificial interferents, including previously utilized tester strains. Recent work has shown that

28 genomic insertion of short identification tags, called "barcodes" here, allows detection of

29 chromosomally tagged strains by real-time PCR. Manual design of these barcodes is feasible for

30 small sets, but expansion of the technique to larger pools of distinct and well-functioning assays

31 would be significantly aided by software-guided design.

33 Results: Here we introduce barCoder, a bioinformatics tool that facilitates the process of creating

34 sets of uniquely identifiable barcoded strains. barCoder utilizes the genomic sequence of the

35 target strain and a set of user-specified PCR parameters to generate a list of suggested barcode

36 "modules" that consist of binding sites for primers and probes and appropriate spacer sequences.

37 Each module is designed to yield optimal PCR amplification and unique identification. Optimal

38 amplification includes metrics such as ideal $\mathrm{T}_{\mathrm{m}}$ and $\mathrm{G}+\mathrm{C}$-content, appropriate spacing, and

39 minimal stem-loop formation; unique identification includes low BLAST hits against the target

40 organism, previously generated barcode modules, and databases, such as NCBI. We tested the

41 ability of our algorithm to suggest appropriate barcodes by generating 12 modules for Bacillus

42 thuringiensis serovar kurstaki, a simulant for the potential biowarfare agent Bacillus anthracis,

43 and three each for other potential target organisms with variable $\mathrm{G}+\mathrm{C}$ content. Real-time PCR

44 detection assays directed at barcodes were specific and yielded minimal cross-reactivity with a

45 panel of near-neighbor and potential contaminant materials. 
47 Conclusions: The barCoder algorithm facilitates the generation of barcoded biological simulants

48 by (a) eliminating the task of creating modules by hand, (b) minimizing optimization of PCR

49 assays, and (c) reducing effort wasted on non-unique barcode modules.

51 Keywords: DNA barcodes, genetic barcoding, genome tagging, tagged strains, microbial

52 forensics, qPCR detection

\section{Background:}

55 Developing an understanding of organisms in their natural ecological niches requires the ability

56 to measure the dynamic interaction with their environment, either at the level of the individual or

57 at population scales. For metazoa, a number of approaches have been utilized to track

58 individuals of a species, including simple bands or markings conferring unique identifiers,

59 Passive Integrated Transponders (PITs), telemetry devices, and biologgers (1, 2). These

60 approaches are limited to large organisms, as they require either direct visual inspection or

61 electronic devices that can be attached by physical means to the body of the organism in

62 question. As the field of environmental microbiology continues to mature, novel tools to

63 facilitate "tag and release" studies are critical to understanding microbial interactions within

64 existing environmental niches or in the context of introduction into new environments. Early

65 efforts to track environmental fate of genetically modified organisms in field releases utilized

66 fluorescently or metabolically marked strains of Pseudomonas putida $(3,4)$ and P. fluorescens

67 (5, 6). Likewise, spontaneous rifampicin-resistant mutants have been used to track establishment

68 and persistence of introduced isolates in field trials (7). However, conventional selectable,

69 chromogenic, or fluorescent markers carry metabolic costs that can compromise the carrier 
70 strain's fitness in resource-constrained environments (8), revealing the need for phenotypically

71 neutral, non-coding, genomic insertions that can differentiate introduced strains from native

72 flora.

74 The development of DNA synthesis chemistry, microarray technology, quantitative PCR (qPCR)

75 and high-throughput sequencing resulted in the development of several important capabilities

76 and tagging approaches. Early studies used transposons containing short synthetic barcodes to

77 identify virulence factors in several organisms $(9,10)$. As oligonucleotide synthesis technology

78 became more sophisticated and costs decreased, longer tags could be produced, resulting in the

79 use of tagged strains to study the spatiotemporal dispersion in systems otherwise unamenable to

80 tracking. In particular, significant work has been done to understand the details of stochastic

81 dynamics of Salmonella infections by monitoring the relative quantities of tagged strains in

82 different locations within the host (11-13). These tagged strains, known as Wild-type Isogenic

83 Tagged Strains (WITS), contain short, unique sequences inserted into the genome to allow

84 quantitation by qPCR (11). Similar work has been done to study population dynamics during

85 infection for several other bacterial and viral pathogens (13-20).

87 The ability to track the fate of microbes introduced into an environment is also of interest to the

88 biodefense research community. Spores of Bacillus anthracis, the causative agent of anthrax,

89 were used in the high-profile 2001 anthrax mail attacks and were historically weaponized by

90 both the United States and Russia on large scales (21). An important angle for preparedness

91 against a potential attack includes an understanding of how spores released into the environment

92 might disperse, persist, and migrate. The release of live B. anthracis spores (and indeed, even of 
93 attenuated strains) in an outdoor test is impossible due to public health concerns. Instead, close

94 biological relatives are used as simulants. In the case of $B$. anthracis, recent work has used

95 Bacillus thuringiensis serovar kurstaki (Btk) due to its similar physiological and biochemical

96 properties (22-26). Yet, even with the use of an adequate simulant, repeated dispersion testing

97 on the same test site is problematic due to a need to distinguish between past and present testing,

98 especially for a ubiquitous environmental bacterium such as Btk that is also in widespread use as

99 a commercial biopesticide (27-30). In addition, the problem of "signature erosion" has

100 diminished the utility of endogenous genomic signatures as detection tools as the diversity of

101 sequence data in public databases has exploded $(31,32)$.

103 To overcome these challenges, we previously inserted unique genetic barcodes designed to 104 enable rapid detection by qPCR into the Btk genome (24) and subsequently tested the system in a 105 field release (23). These strains were successfully detected in field samples using the qPCR 106 assays, but, like the earlier WITS strains, the strains constructed for our field release (23) did not 107 exploit the full ability of bioinformatics and synthetic biology that has become available. Most 108 notably, each of the tags required its own specific PCR assay conditions, which makes scaling up 109 to larger numbers of barcodes prohibitive. In this work, we have built upon our previous work 110 by developing an algorithm, called barCoder, to generate barcode sequences that are unique 111 amongst a pool of barcoded strains and require minimal development of qPCR assays. The 112 algorithm also provides numerous features to minimize experimental troubleshooting efforts and 113 customize amplicon properties. Here, we present the algorithm, as well as experimental 114 validation of its ability to generate a potentially unlimited pool of highly diverse DNA barcodes, 115 each with its own specific qPCR assay. 


\section{Results:}

118 Barcode design

119 Two major types of qPCR assays exist: assays based on intercalating dyes (e.g. SYBR Green)

120 responsive only to double-stranded DNA, and assays based on $3 \square$-exonuclease degradation of

121 probe sequences effecting a signal unquenching (referred to herein as TaqMan for the probes

122 used). In terms of assay design, both require a forward and reverse primer with similar

123 constraints such as amplicon length, $\mathrm{T}_{\mathrm{m}}, \mathrm{G}+\mathrm{C}$-content, $\mathrm{G}+\mathrm{C}$-clamp, potential secondary

124 structure, and primer dimer formation. The primary design difference between the qPCR assay

125 types is a third probe sequence required only for TaqMan assays with its own recommended

126 design guidelines. Thus, in general, the same primer set can be used for either approach with the

127 same barcode (Fig 1A). From the perspective of qPCR assay design, the remaining spacer

128 sequences between primers/probe are largely immaterial other than to meet ideal amplicon size

129 targets, and therefore can be generated randomly, with constraints (see Algorithm design).

\section{Algorithm design}

132 The barCoder algorithm workflow is depicted in Figure 1B. The algorithm starts by generating 133 random primers to meet PCR-related specifications. Unlike typical primer design where primer 134 sequences are constrained by an existing sequence of interest, here there is almost complete 135 freedom to design primers that have ideal PCR properties. Using an approximate $\mathrm{T}_{\mathrm{m}}$ formula (see

136 Methods), the number of A's+T's and G's+C's needed to satisfy the specified $\mathrm{T}_{\mathrm{m}}$ value can be

137 calculated for primers within user-adjustable constraints on length and G+C content. From this 138 set, a sequence meeting these constraints is randomly generated and screened for several PCR- 
bioRxiv preprint doi: https://doi.org/10.1101/2020.01.06.896035; this version posted January 7, 2020. The copyright holder for this preprint (which was not certified by peer review) is the author/funder, who has granted bioRxiv a license to display the preprint in perpetuity. It is made available under aCC-BY-NC-ND 4.0 International license.

139 related properties, such as maximum homopolymer repeats and secondary structure (Table 1). If

140 any requirements are not met, the sequence is discarded and a new sequence generated. 
141 Table 1. Algorithm parameters and default values for primer design.

\begin{tabular}{|c|c|c|c|c|}
\hline \multirow[b]{2}{*}{ Purpose } & \multirow[b]{2}{*}{ Parameter Purpose } & \multicolumn{3}{|c|}{ Values used in this study $^{a}$} \\
\hline & & $\begin{array}{l}\text { Forward } \\
\text { Primer }\end{array}$ & $\begin{array}{l}\text { Reverse } \\
\text { Primer }\end{array}$ & Probe \\
\hline \multirow[t]{6}{*}{ Primer Constraints } & Minimum Length (bases) & 20 & 20 & 20 \\
\hline & Maximum Length (bases) & 24 & 24 & 30 \\
\hline & Minimum $\operatorname{Tm}\left({ }^{\circ} \mathrm{C}\right)$ & 58 & 58 & 68 \\
\hline & Maximum $\operatorname{Tm}\left({ }^{\circ} \mathrm{C}\right)$ & 60 & 60 & 70 \\
\hline & Minimum G+C Content (\%) & 40 & 40 & 40 \\
\hline & Maximum G+C Content $(\%)$ & 60 & 60 & 60 \\
\hline \multirow[t]{5}{*}{ Primer Checks } & Maximum G Homopolymer Length (bases) & 4 & 4 & 4 \\
\hline & $\begin{array}{l}\text { Maximum A/T/C Homopolymer Length } \\
\text { (bases) }\end{array}$ & 3 & 3 & 3 \\
\hline & No G on $5 \square$ end of probe & $\mathrm{n} / \mathrm{a}$ & $\mathrm{n} / \mathrm{a}$ & $\mathrm{Y} / \mathrm{N}$ \\
\hline & $>2 \mathrm{G}+\mathrm{C}$ 's in last $5 \mathrm{bps}$ of $3 \square$ end of primers & $\mathrm{Y} / \mathrm{N}$ & $\mathrm{Y} / \mathrm{N}$ & $\mathrm{n} / \mathrm{a}$ \\
\hline & Any start codons present? & $\mathrm{Y} / \mathrm{N}$ & $\mathrm{Y} / \mathrm{N}$ & $\mathrm{Y} / \mathrm{N}$ \\
\hline \multirow[t]{5}{*}{ Primer Stem-loops } & Minimum Stem-loop Hydrogen Bonds & 14 & 14 & 14 \\
\hline & Minimum Stem-loop Palindrome Length & 5 & 5 & 5 \\
\hline & Maximum Stem-loop Palindrome Length & 100 & 100 & 100 \\
\hline & Maximum Stem-loop Gap Size & 11 & 11 & 11 \\
\hline & Maximum Stem-loop Mismatches & 1 & 1 & 1 \\
\hline
\end{tabular}

$142{ }^{a}$ Entries of "Y/N" represent checks that are pass/fail and thus do not have a parameter value per-

143 se.

145 A sequence that meets PCR restrictions is then tested for uniqueness. First, the sequence is

146 compared to a list of other primers, which includes any primers already generated locally by the

147 algorithm and an optional user-provided list of other primer sets of interest. Sequence "matches"

148 are determined by comparing raw BLAST scores divided by the raw score of a perfect match to a

149 user-adjustable threshold. If the sequence matches any existing primers above the threshold, the

150 sequence is discarded and the process restarted. Second, the genome of the organism targeted for

151 insertion is scanned for similar sequences by BLAST. Similarly, a set of additional genome

152 sequences of organisms that may be likely to be present in a sample, such as common

153 environmental background species or human, are scanned. Finally, the entire NCBI database is

154 optionally scanned for similar sequences. A separate threshold for discarding a candidate 
155 sequence based on these BLAST results can be customized by the user, allowing more or less

156 stringent criteria depending on project demands and acceptable CPU time in the case of very

157 strict thresholds. Values used in this study are shown in Table 2.

159 Table 2. Algorithm parameters and default values other than for primer design.

\begin{tabular}{|l|l|c|}
\hline \multicolumn{1}{|c|}{ Purpose } & \multicolumn{1}{|c|}{ Parameter Purpose } & $\begin{array}{c}\text { Values used in } \\
\text { this study }\end{array}$ \\
\hline \multirow{2}{*}{ BLAST } & Threshold $^{a}$ to reject near-matches (primers) & 0.85 \\
\cline { 2 - 3 } & Threshold $^{a}$ to reject near-matches (genomes) & 0.65 \\
\hline \multirow{2}{*}{ Spacer Size } & $\begin{array}{l}\text { Spacer Size (distance between forward/reverse } \\
\text { primers, not inclusive) }\end{array}$ & 100 \\
\hline \multirow{3}{*}{ Spacer Stem-loops } & Minimum Stem-loop Hydrogen Bonds & 14 \\
\cline { 2 - 3 } & Minimum Stem-loop Palindrome Length & 10 \\
\cline { 2 - 3 } & Maximum Stem-loop Palindrome Length & 80 \\
\cline { 2 - 3 } & Maximum Stem-loop Gap Size & 11 \\
\cline { 2 - 3 } & Maximum Stem-loop Mismatches & 1 \\
\hline
\end{tabular}

${ }^{a}$ See text for explanation of threshold calculation.

162 A sequence that meets all PCR and uniqueness requirements is accepted for use in the barcode.

163 The algorithm cycles through this process to create each primer and the probe sequence, each

164 with its own set of requirement parameters. Optionally, the forward primer can be set as constant

165 for all barcodes in a given project. Once all three primer/probe sequences for a barcode have

166 been generated, the spacer sequences are randomly generated such that the total length

167 requirement is met and the $\mathrm{G}+\mathrm{C}$ content of the full barcode matches the $\mathrm{G}+\mathrm{C}$ content of the

168 target organism. The final check scans for potential stem-loop structures in the barcode to limit

169 challenges during genome insertion and during amplification of the sequence. Failing this check

170 triggers regeneration of the spacer sequences.

171

172 Experimental Validation 
173 The barCoder algorithm was used to generate an initial set of 21 barcodes and corresponding

174 qPCR detection primer/probe sets (sequences listed in Tables S1 and S2 in Additional file 1).

175 Twelve of these barcodes were designed for B. thuringiensis serovar kurstaki (Btk), a surrogate

176 for the biothreat agent $B$. anthracis with low-G+C content $(35 \%$, (33)). To demonstrate the

177 utility of the barCoder algorithm to create barcodes for other organisms, including those with

178 different G+C compositions, three barcodes each were designed for potential use in Burkholderia

179 pseudomallei 1026b, (68\% G+C content), Yersinia pestis CO92 (47\%), and Clostridium

180 botulinum Hall A (28\%) (34-36).

181

182 Assay conditions for barcode Btk1 in the pIDTSMART-AMP plasmid backbone were optimized

183 and subsequently standard curves were generated for all 21 TaqMan qPCR assays using the same

184 conditions (Fig. 2 and Fig. S1 in Additional file 1). All of the assays of the barcodes in plasmids

185 performed well with qPCR efficiencies ranging from $81.1 \%$ to $100.0 \%$, strong linear

186 relationships $\left(\mathrm{R}^{2}>0.99\right)$, and no false positive results (Table 3). Limits of detection (LODs)

187 were all below 50 copies (the lowest plasmid concentration tested), except for barcode Btk6,

188 where the LOD was below 500 copies (Table 3). Select barcodes were also markerlessly

189 incorporated into the chromosomes of potential target organisms: barcode Btk1 was integrated

190 into both Btk and B. anthracis Sterne, and barcode Yp1 was inserted into a pgm ${ }^{-}$derivative of $Y$.

191 pestis CO92. Again, standard curves were generated for the TaqMan assays under the same

192 conditions (Fig. 2). Assays using chromosomally-barcoded strains had efficiencies within the

193 range observed for barcodes residing in plasmids ( $86.5 \%$ to $96.5 \%), \mathrm{R}^{2}$ values above 0.99 , and

194 no false positives (Table 3). LODs were calculated as less than 15 copies and less than 2 copies

195 for barcode Btk1 in the chromosomes of Btk and B. anthracis Sterne, respectively, and less than 

approximate as lower concentrations and Poisson distribution effects at low copy numbers were not thoroughly interrogated.

Table 3. Evaluation of qPCR assays from generated standard curves.

\begin{tabular}{|c|c|c|c|c|c|}
\hline Barcode & $\begin{array}{l}\text { Template } \\
\text { DNA }\end{array}$ & Linearity $\left(\mathbf{R}^{2}\right)$ & LOD (Copies) & False +'s & $\begin{array}{c}\text { Efficiency } \\
(\%)\end{array}$ \\
\hline \multirow{3}{*}{ Btk1 } & Plasmid & 0.9990 & $<50$ & $0 / 3$ & 92.5 \\
\hline & Genomic (Btk) & 0.9969 & $<15$ & $0 / 3$ & 91.1 \\
\hline & $\begin{array}{l}\text { Genomic (Ba } \\
\text { Sterne) }\end{array}$ & 0.9919 & $<2$ & $0 / 3$ & 96.5 \\
\hline Btk2 & Plasmid & 0.9984 & $<50$ & $0 / 3$ & 83.5 \\
\hline Btk3 & Plasmid & 0.9991 & $<50$ & $0 / 3$ & 98.9 \\
\hline Btk4 & Plasmid & 0.9976 & $<50$ & $0 / 3$ & 94.3 \\
\hline Btk5 & Plasmid & 0.9978 & $<50$ & $0 / 3$ & 89.9 \\
\hline Btk6 & Plasmid & 0.9996 & $<500$ & $0 / 3$ & 90.4 \\
\hline Btk7 & Plasmid & 0.9993 & $<50$ & $0 / 3$ & 84.0 \\
\hline Btk8 & Plasmid & 0.9979 & $<50$ & $0 / 3$ & 89.8 \\
\hline Btk9 & Plasmid & 0.9994 & $<50$ & $0 / 3$ & 81.1 \\
\hline Btk10 & Plasmid & 0.9985 & $<50$ & $0 / 3$ & 89.9 \\
\hline Btk11 & Plasmid & 0.9990 & $<50$ & $0 / 3$ & 90.6 \\
\hline Btk12 & Plasmid & 0.9985 & $<50$ & $0 / 3$ & 82.5 \\
\hline Bp1 & Plasmid & 0.9996 & $<50$ & $0 / 3$ & 93.0 \\
\hline Bp2 & Plasmid & 0.9996 & $<50$ & $0 / 3$ & 100.0 \\
\hline Bp3 & Plasmid & 0.9992 & $<50$ & $0 / 3$ & 92.4 \\
\hline Cbot1 & Plasmid & 0.9983 & $<50$ & $0 / 3$ & 95.1 \\
\hline Cbot2 & Plasmid & 0.9982 & $<50$ & $0 / 3$ & 91.6 \\
\hline Cbot3 & Plasmid & 0.9990 & $<50$ & $0 / 3$ & 86.1 \\
\hline \multirow{2}{*}{ Yp1 } & Plasmid & 0.9988 & $<50$ & $0 / 3$ & 94.6 \\
\hline & Genomic & 1.0000 & $<25$ & $0 / 3$ & 86.5 \\
\hline Yp2 & Plasmid & 0.9979 & $<50$ & $0 / 3$ & 96.8 \\
\hline Yp3 & Plasmid & 0.9997 & $<50$ & $0 / 3$ & 95.0 \\
\hline
\end{tabular}

202 To test the specificity of the TaqMan qPCR assays for the corresponding barcode, each of the 12

203 Btk assays were tested against all 12 Btk barcodes in plasmids. This cross-reactivity panel

204 showed unique amplification of each Btk barcode with its cognate primer/probe set (Fig. 3). The

205 TaqMan qPCR assay for barcode Btk1 was also tested against a panel of potential pathogens and 
206 environmental organisms (Table 4). Reactions containing the Btk strain with barcode Btk1

207 inserted in the chromosome, either alone or in the presence of an environmental matrix (DNA

208 from a mock microbial community or DNA extracted from soil) showed robust positive results,

209 while the Btk1 qPCR assay did not cross-react with any of the potential contaminants. 
211 Table 4. Cross-reactivity of the barcode Btk1 qPCR assay against a panel of potential pathogens

212 and environmental contaminants.

\begin{tabular}{|c|c|}
\hline DNA Template ${ }^{a}$ & $\mathbf{C t}^{b}$ \\
\hline Bacillus anthracis Ames35 & ND \\
\hline Bacillus anthracis Sterne 34F2 & ND \\
\hline Bacillus cereus Gibson 971 & ND \\
\hline Bacillus licheniformis Gibson 46 & ND \\
\hline Bacillus megaterium Ford 19 & ND \\
\hline Bacillus sphaericus Ford 25 & ND \\
\hline Bacillus subtilis subsp. subtilis 168 & ND \\
\hline Bacillus thuringiensis serovar kurstaki HD-1 & ND \\
\hline Bacillus thuringiensis subsp. konkukian 97-27 & ND \\
\hline Burkholderia pseudomallei 1026b & ND \\
\hline Clostridium perfringens WAL-14572 & ND \\
\hline Escherichia coli EDL933 & ND \\
\hline Francisella tularensis subsp. tularensis SCHU S4 & ND \\
\hline Micrococcus luteus SK58 & ND \\
\hline Neisseria meningitidis 9506 & ND \\
\hline Pseudomonas sp.2_1_26 & ND \\
\hline Salmonella enterica subsp. enterica LT2 & ND \\
\hline Staphylococcus aureus TCH1516 & ND \\
\hline Staphylococcus epidermidis SK135 & ND \\
\hline Streptococcus pneumoniae TCH8431 & ND \\
\hline Vibrio cholerae 395 & ND \\
\hline Yersinia pestis $\mathrm{CO} 92$ & ND \\
\hline Barcode Btk1 in Bacillus thuringiensis serovar kurstaki HD-1 & 23.927 \\
\hline Microbial Mock Community B (Even, High Concentration) & ND \\
\hline $\begin{array}{l}\text { Microbial Mock Community B (Even, High Concentration) + Barcode Btk1 in } \\
\text { Bacillus thuringiensis serovar kurstaki HD-1 }\end{array}$ & 23.725 \\
\hline Soil DNA extract & ND \\
\hline Soil DNA extract + Barcode Btk1 in Bacillus thuringiensis serovar kurstaki HD-1 & 23.969 \\
\hline Negative control $\left(\mathrm{H}_{2} \mathrm{O}\right)$ & ND \\
\hline
\end{tabular}

$213{ }^{a}$ PCR reactions contained $1 \mathrm{ng}$ of each DNA template indicated.

$214{ }^{b}$ Threshold cycle $(\mathrm{Ct})$ values are the median of three replicates; ND, Not determinable.

216 Discussion: 
217 qPCR has become a standard technique for detection of microorganisms in the environment and

218 for diagnosis of infection (37), and, as such, is an attractive detection technology that also allows

219 a rapid evaluation of the relative abundance of a known microorganism within a sample.

220 However, when conducting environmental fate studies, for example, these assays must

221 discriminate from the endogenous or native microflora, which may be uncharacterized and

222 present signatures similar to or cross-reactive with the signature selected for detection of the

223 experimental strain. We sought in this report to utilize a bioinformatics strategy to generate

224 specific amplicons that require minimal assay optimization and could be introduced into

225 organisms with minimal to no cross-reactivity with environmental and microbial signatures.

227 Our approach to developing unique qPCR-compatible barcodes expanded upon our previous 228 work, in which we appropriated synthetic signatures from published microarrays and developed

229 PCR assays based on the unique sequences generated both by the tags themselves and by their 230 insertion into the genome (24). Because those sequences were not designed de novo for use in

231 PCR detection assays, we relied on the presence of a chromosomal primer binding site and the

232 single synthetic sequence to generate suitable amplicons. As a result, considerable optimization

233 of the assay conditions and primer sequences was necessary during the development of those

234 strains, and the assay conditions for each tag required slightly different optimal conditions for 235 detection. This situation was judged as suboptimal for the development of a more diverse panel 236 of barcodes, as new assays would need to be developed for each new sequence.

238 We therefore sought to develop an algorithm that would enable the high-throughput generation

239 of amplicon sequences that could use a single PCR assay condition, and in which relative 
240 proportion of each strain could be compared in a single test, e.g. across a single microplate. The

241 assays would need to be specific to each barcode, and would need to be comparably sensitive

242 with equivalent limits of detection. Using TaqMan ${ }^{\mathrm{TM}}$ qPCR chemistry and a stringent

243 bioinformatic screening algorithm, we generated a panel of unique primer/probe combinations

244 that exhibited the desired combination of selectivity, specificity, and sensitivity. Using

245 conventional plasmids containing the barcodes as templates for the development of the assays,

246 we demonstrate strong performance in linearity of response, sensitivity, and efficiency across 21

247 assays using conditions optimized for a single assay. No cross-reactivity was observed across a

248 panel of 12 of these assays. We note that the odds of randomly generating a barcode that would

249 react with a natural sequence is vanishingly small as three $20+$ bp primer sequences would need

250 to be closely matched in the correct orientation $\left(>10^{36}\right.$ possible sequences) with spacing

251 appropriate for PCR amplification; nonetheless, sequences are screened for uniqueness to further

252 minimize this possibility. Inserting two of the barcodes into three different genomes, we

253 observed conserved performance compared to plasmid assays and LODs below 25 copies, which

254 we believe to be conservative due to Poisson distribution effects at low copy numbers.

256 Our barcodes have a number of potential applications. Marking strains with unique artificial

257 signatures could aid in protecting intellectual property, particularly for production strains whose

258 development has required significant investment in metabolic and/or genetic optimization,

259 perhaps in combination with other techniques such as DNA steganography (38). While not as

260 information-rich as longer steganographic tags or watermarks $(39,40)$, qPCR barcodes have the

261 advantage of not requiring further sequencing and informatic analysis to detect and/or verify

262 their presence; they must simply be amplified using appropriate primers and probes. In one 
263 scenario, a set of barcodes could be inserted at defined intervals throughout a large DNA

264 molecule used for information storage, and utilized to provide a preliminary indicator of the

265 stability of the archive prior to full sequencing.

267 These sequences and their associated assays might also find use in forensic applications. In

268 particular, one might imagine their use as molecular taggants that could be spiked into samples

269 by field technicians, and their detection in DNA samples by the reference laboratories would

270 serve to verify the origin of the sample. In a similar vein, these same tools could be used in the

271 future for downstream attribution of accidental or deliberate release of organisms (41). Select

272 agent strains, in particular, could be tagged, distributed to end-user communities, and then any

273 material from the scene of a biocrime could be rapidly amplified using the library of primers and

274 probes, enabling the rapid focus of investigative resources on those potential sources, while

275 excluding the majority of the research laboratories that possess variants containing other

276 barcodes. Any mechanism by which artificial genetic diversity can be introduced into the largely

277 clonal populations of laboratory strains would be useful as all known acts of bioterrorism to date

278 have utilized common laboratory strains (e.g. B. anthracis Ames Ancestor in the 2001 U.S.

279 Mail/Amerithrax case; S. enterica serovar typhimurium 14028s in the Rajneeshi cult attacks of

280 1984) (42). In the case of the Amerithrax samples, discriminating between samples present in

281 these laboratories relied on presence of several spontaneous mutants whose discovery and

282 characterization required astute microbiologists and what were at the time Herculean sequencing

283 efforts $(43,44)$. The deliberate incorporation of end-user-specific sequences into such

284 commonly available strains could immeasurably speed identification of potential originating

285 laboratories, would help investigators narrow their focus to a subset of potential sources, and 
286 would help exclude uninvolved laboratories working on similar research as potential sources.

287 Furthermore, the presence of such signatures (and the knowledge that significant additional

288 effort would be required to disguise the source of a sample) could deter potential malefactors

289 within those laboratories even if the location, sequence, and properties of the sequence were

290 known.

292 Conclusions:

293 To our knowledge, barCoder represents the first completely in silico method for generating both

294 a synthetic target for qPCR and the primers/probe to amplify the target, and optimal assay

295 conditions for detection of a diverse range of barcodes. We demonstrated that generated

296 barcodes all perform well under a single set of assay conditions and show no cross-reactivity

297 with themselves or environmental contaminants. Insertion of the barcodes into the genomes of

298 three organisms of interest maintained the key properties of the barcodes. We anticipate

299 barCoder finding utility in applications such as environmental fate studies, intellectual property,

300 and microbial forensics.

301

302 Methods

303 Algorithm Design

304 All software was written in Perl. G+C and A+T constraints were calculated using the following 305 formula:

$$
T_{m}=69.4+\frac{41 *\left(n_{G C}-16.4\right)}{n_{\text {total }}}
$$

306 where $n_{G C}$ is the number of $\mathrm{G}$ or $\mathrm{C}$ bases and $n_{\text {total }}$ is the length of the primer. 
307 Most bioinformatics functions were implemented using existing BioPerl modules. EMBOSS

308 software, called by BioPerl, was used to predict stem-loop structures. All BLAST runs used

309 default BioPerl parameters. Software is available on GitHub

310 (https://github.com/ECBCgit/Barcoder).

312 barCoder-designed elements and sources of DNA

313 All barcode modules were designed using the barCoder algorithm using the values listed in

314 Tables 1 and 2. For the BLAST step against the target organism genome sequence, the following

315 NCBI accession numbers were used: B. thuringiensis serovar kurstaki, NZ_CP010005.1; B.

316 pseudomallei 1026b, NC_017831 (chromosome 1) and NC_017832 (chromosome 2); C.

317 botulinum Hall A, NC_009495.1; Y. pestis CO92, NC_003143. All barcodes, primers, and

318 probes were obtained from Integrated DNA Technologies, Inc. (IDT, Coralville, IA) and their

319 sequences listed in Tables S1 and S2 in Additional file 1. Btk barcodes were designed with

320 SacI/NheI restriction sites flanking the synthetic elements to facilitate later subcloning.

321 Barcodes were received as "minigenes" inserted in the pIDTSMART-AMP plasmid backbone

322 and were propagated in NEB® 5-alpha E. coli (New England Biolabs, Inc., Ipswich, MA) on

323 Luria-Bertani (LB) agar and in LB broth with $100 \mu \mathrm{g} / \mathrm{ml}$ ampicillin at $37^{\circ} \mathrm{C}$. Plasmid DNA was

324 isolated using the QIAprep Spin Miniprep Kit (Qiagen, Hilden, Germany). DNA probes were

325 ordered as PrimeTime double-quenched qPCR probes containing the $5 \square$ FAM fluorophore, $3 \square$

326 Iowa Black FQ quencher, and internal ZEN quencher. The sources of the DNA used for the

327 cross-reactivity panel of pathogenic and environmental organisms are given in Table S3 in

328 Additional file 1. 
331 All qPCR experiments were run on an Applied Biosystems 7900HT Real-Time PCR System

332 (Applied Biosystems, Foster City, CA) using Applied Biosystems MicroAmp optical 384-well

333 reaction plates (catalog number 4309849) sealed with Applied Biosystems MicroAmp optical

334 adhesive film (catalog number 4311971). Optimized $20 \mu \mathrm{L}$ reactions included Applied

335 Biosystems TaqMan® Universal PCR Master Mix (catalog number 4304437), forward and 336 reverse primers each at a final concentration of $900 \mathrm{nM}$, DNA probe at a final concentration of

$337250 \mathrm{nM}, 1 \mu \mathrm{L}$ DNA template at the indicated concentration, and nuclease-free water. TaqMan

338 assays used the following thermocycler protocol: 1 cycle of $50^{\circ} \mathrm{C}$ for $2 \mathrm{~min}, 1$ cycle of $95^{\circ} \mathrm{C}$ for

$33910 \mathrm{~min}$, and 40 cycles of $95^{\circ} \mathrm{C}$ for $15 \mathrm{sec}$ and $55^{\circ} \mathrm{C}$ for $1 \mathrm{~min}$. The standard curve properties of

340 each assay were assessed by performing 10-fold serial dilutions of the template DNA in

341 nuclease-free water. Efficiency and linearity $\left(\mathrm{R}^{2}\right)$ values for each $\mathrm{qPCR}$ standard curve were

342 calculated using the median $\mathrm{Ct}$ of three replicates for each template DNA dilution. Data points

343 corresponding to the highest amount of template DNA tested $\left(10^{-8} \mathrm{~g}\right.$ for genomic DNA, $10^{-9} \mathrm{~g}$

344 for plasmid DNA) were omitted from these analyses in all cases as the Ct values tended to be

345 non-linear with the other data points of the standard curve. LODs were conservatively estimated

346 using the lowest amount of template DNA tested that produced a $\mathrm{Ct}$ value $<40$ for all three

347 replicates.

349 Construction of genomically-barcoded strains

350 B. thuringiensis and B. anthracis strains were routinely cultured on Brain Heart Infusion (BHI)

351 agar and in $\mathrm{BHI}$ broth at $30^{\circ} \mathrm{C}$ (B. thuringiensis) or $37^{\circ} \mathrm{C}$ (B. anthracis). Unless otherwise

352 indicated, $Y$. pestis strains were grown on BHI agar or Tryptic Soy Agar (TSA), and in BHI broth 
353 at $28-30^{\circ} \mathrm{C}$. Genomic DNA was extracted using the UltraClean Microbial DNA Isolation Kit

354 (MOBIO Laboratories, Inc., Carlsbad, CA). Barcode Btk1 was selected to construct a strain in

355 which the barcode was markerlessly incorporated into the chromosome of B. thuringiensis

356 serovar kurstaki HD-1 (24, 33) (obtained from the DoD Unified Culture Collection

357 (https://www.usamriid.army.mil/ucc/)). The insertion was generated at the same locus that was

358 identified and modified in our previous report (within Target 1, (24)). This corresponds to an

359 insertion between positions 4,834,064 and 4,834,065 of RefSeq accession number

360 NZ_CP010005.1. Plasmid pRP1028-T1-PL (sequence provided in Additional file 2), a

361 derivative of pRP1028 (45), was designed specifically for incorporating synthetic elements

362 within this target region of the Btk chromosome and was synthesized by DNA2.0 (Menlo Park,

363 CA). Plasmid pRP1028-T1-PL contains 1,550 bp of DNA homologous to the Btk chromosomal

364 insertion region between the pRP1028 HindIII and BamHI sites, as well as a 36-bp polylinker

365 within the homology region. Following digestion of plasmid pIDTSMART-AMP:Barcode Btk1

366 with SacI and NheI, the Btk1 barcode was gel extracted (QIAquick Gel Extraction Kit, Qiagen,

367 Hilden, Germany) and ligated with pRP1028-T1-PL that had been digested with the same

368 restriction enzymes. This pRP1028-T1-PL derivative containing barcode Btk1 was introduced

369 into Btk, and the barcode was incorporated into the chromosome using the markerless allelic

370 exchange strategy described previously (45). Successful barcode integration into the Btk

371 chromosome was verified by PCR amplification of the target locus and SacI/NheI digestion of

372 the resulting amplicon. Construction of a strain of $B$. anthracis Sterne 34F2 with barcode Btk1

373 in the chromosome was previously published (46). 
375 For construction of a strain of $Y$. pestis CO92 $\mathrm{pgm}^{-}$with barcode Yp1 markerlessly inserted in

376 the chromosome, the locus between the convergently transcribed genes YPO0388 and

377 YPO0392a (RefSeq accession number NC_003143.1) was selected using rules adopted from

378 Buckley et al. (24), in combination with the PATRIC database (47) and available transcriptome

379 sequencing (RNA-seq) data (SRA accession numbers SRR1013703, SRR1013704,

380 SRR1013705, SRR1041589), to identify a potentially neutral insertion region. The barcode was

381 then inserted into the chromosome between positions 406,742 and 406,743 via the method

382 described by Sun et al. (48), which utilizes $\lambda$ Red recombination and $s a c B$ counterselection.

383 Briefly, plasmid pKD46 (CGSC \#7739, (48)) containing the genes for $\lambda$ Red recombination was

384 electroporated into a strain of $Y$. pestis CO92 $\mathrm{pgm}^{-}$(strain R88, Robert Perry, University of

385 Kentucky). A linear DNA fragment containing a cat-sacB cassette flanked by DNA homologous

386 to the $Y$. pestis chromosomal insertion region was electroporated into this pKD46-containing

387 strain of $Y$. pestis, and successful integrants were selected on media containing chloramphenicol.

388 Following electroporation with a linear DNA fragment containing barcode Yp1 flanked by

389 homologous DNA and selection on media containing sucrose, the cat-sacB cassette in the

390 chromosome was replaced with the barcode. The resulting strain was subsequently cured of

391 pKD46, and successful barcode insertion was verified by PCR amplification and sequencing.

392 Whole-genome sequencing (MiSeq, Illumina) was also performed to confirm the absence of off-

393 target modifications. Primers used to construct this barcoded strain of $Y$. pestis are listed in

394 Table S4 in Additional file 1. To generate the cat-sacB cassette, the cat gene was PCR amplified

395 from plasmid pKD3 (CGSC \#7631, (49)) with primers \#1 and \#2, and the sacB gene was PCR

396 amplified from plasmid p88171 (synthesized plasmid with pJ207 backbone and sacB gene from

397 Bacillus subtilis, DNA 2.0, Menlo Park, CA) with primers \#3 and \#4. The two PCR amplicons 
were purified (QIAquick PCR Purification Kit, Qiagen, Hilden, Germany) and joined together by overlap extension PCR (50) using primers \#1 and \#4. The cat-sacB cassette was gel extracted and cloned between the SacI and BamHI sites of pUC19 to create plasmid pCBV4. To construct

401 the $c a t-s a c B$ cassette flanked by homologous DNA, the cat-sacB cassette was PCR amplified 402 from pCBV4 with primers \#5 and \#6. Approximately 500 bp flanking each side of the barcode

403 insertion point were separately PCR amplified from the Y. pestis CO92 pgm $^{-}$chromosome;

404 primers \#7 and \#8 were used to amplify upstream DNA, and primers \#9 and \#10 were used to 405 amplify downstream DNA. The three purified PCR amplicons (up flanking region, $c a t-s a c B$ 406 cassette, and down flanking region) were joined together by overlap extension PCR (50) using 407 primers \#7 and \#10, and the resulting amplicon was gel extracted and cloned into the 408 pCR ${ }^{\mathrm{TM}} 4 \mathrm{Blunt}-\mathrm{TOPO}{ }^{\circledR}$ vector (Invitrogen, Carlsbad, CA) to generate plasmid pCBV6. The 409 linear DNA fragment containing the cat-sacB cassette flanked on both sides by Y. pestis CO92 $410 \mathrm{pgm}^{-}$DNA was PCR amplified from pCBV6 with primers \#7 and \#10. To create barcode Yp1

411 flanked by homologous DNA, the barcode was PCR amplified from the synthesized plasmid 412 pIDTSMART-AMP:Barcode Yp1 using primers \#11 and \#12. Approximately 500 bp flanking 413 each side of the barcode insertion point were separately PCR amplified from the $Y$. pestis CO92 $414 \mathrm{pgm}^{-}$chromosome; primers \#7 and \#13 were used to amplify upstream DNA, and primers \#10 415 and \#14 were used to amplify downstream DNA. The three purified PCR amplicons (up 416 flanking region, barcode, and down flanking region) were joined by overlap extension PCR (50) 417 using primers \#7 and \#10, and the resulting amplicon was gel extracted and cloned into the 418 pCR ${ }^{\mathrm{TM}} 4 \mathrm{Blunt}-\mathrm{TOPO}{ }^{\circledR}$ vector (Invitrogen, Carlsbad, CA) to generate plasmid pCBV9. The 419 linear DNA fragment containing barcode Yp1 flanked on both sides by Y. pestis CO92 pgm $^{-}$ 420 DNA was PCR amplified from pCBV9 with primers \#7 and \#10. 


\section{Declarations}

424 Ethics approval and consent to participate

425 Not applicable

426

Consent for publication

428 Not applicable

429

430 Availability of data and material

431 All raw data to the level of $\mathrm{Ct}$ values that were generated and analyzed during this study are 432 included in the article and its additional files.

Competing interests

435 The authors declare that they have no competing interests.

$437 \quad$ Funding

438 Funding for this work was provided by the Defense Threat Reduction Agency under project 439 CB3654 and by the Defense Threat Reduction Agency/National Research Council Postdoctoral 440 Research Associateship Program (to CBB). This work is approved for public release. The

441 opinions expressed in this report are those of the authors and do not represent official policy of 442 the United States Government or any of its agencies. 
445 CBB inserted barcodes into B. anthracis and $Y$. pestis, designed and performed qPCR

446 experiments, analyzed data, and wrote the manuscript. MWL developed the algorithm, designed

447 qPCR experiments, analyzed data, and wrote the manuscript. SEK optimized qPCR protocols.

448 TDPG inserted barcode Btk1 into Btk. ATL updated the algorithm for publication. HSG

449 conceived and led the project, analyzed data, and wrote the manuscript.

451 Acknowledgements

452 We thank F. Chris Minion (Iowa State University) for the generous gift of $Y$. pestis strain R88.

453 We also thank Mark Karavis (CCDC CBC) for whole-genome sequencing, and Pierce Roth,

454 Edward Fochler (DCS Corp/CCDC CBC), and Michael Krepps (BrightEdge Investments) for

455 bioinformatics support.

457 Additional Files:

458 Additional file 1.docx: Supplementary Tables and Figure. Tables S1 contains sequences for the

45921 barcodes designed with the barCoder algorithm. Table S2 contains primer and probe 460 sequences for each barcode module. Table S3 provides the sources of DNA used in the cross-

461 reactivity panel shown in Table 4 . Table S4 contains sequences of the primers used to construct

462 the barcoded strain of $Y$. pestis CO92 $\mathrm{pgm}^{-}$. Figure S1 shows additional qPCR standard curves.

464 Additional file 2.gbk: Plasmid pRP1028-T1-PL sequence. This file contains the complete

465 annotated sequence for the plasmid designed for incorporating synthetic elements within the 466 target region of the Btk chromosome. 
468 Additional file 3.xlsx: Standard curve data. This file contains Ct values and calculations used to

469 generate and analyze qPCR standard curves for all 21 barcodes (in the plasmid backbone and

470 inserted in the genome, if applicable).

471

472 Additional file 4.xlsx: Raw data for the cross-reactivity panel of Btk barcodes and qPCR assays.

473 This file contains raw $\mathrm{Ct}$ values from the cross-reactivity panel of the 12 Btk qPCR assays

474 against the 12 Btk barcodes.

475

476 Additional file 5.xlsx: Raw data for the Btk1 qPCR assay cross-reactivity panel. This file

477 contains the raw $\mathrm{Ct}$ values from the cross-reactivity panel of the barcode Btk1 qPCR assay

478 against a panel of potential pathogens and environmental contaminants. 


\section{Figure Legends}

485 Figure 1. Overview of barcode design and algorithm work flow. A) Barcodes consist of two 486 synthetic primer binding sites, a probe annealing site, and two spacer regions. Spacer regions 487 can be adjusted to match the overall $\mathrm{G}+\mathrm{C}$ content of the organism to be barcoded. B) barCoder 488 algorithm work flow.

490 Figure 2. Representative qPCR assay standard curves. Curves were generated using A) barcode 491 Btk1 in the pIDTSMART-AMP plasmid backbone, B) barcode Btk1 inserted into the $B$. 492 thuringiensis kurstaki chromosome, C) barcode Btk1 inserted into the B. anthracis Sterne 493 chromosome, and D) barcode Yp1 inserted into the $Y$. pestis CO92 pgm $^{-}$chromosome. For each 494 standard curve, data from three replicates and a trendline are shown. Standard curves for the 495 remaining barcodes in the plasmid backbone are shown in Figure S1 in Additional file 1.

$496 \square \mathrm{Ct}$ value not determinable for $2 / 3$ replicates.

$497 \square$ Ct value not determinable for $3 / 3$ replicates.

499 Figure 3. Cross-reactivity of the 12 Btk qPCR assays against the 12 Btk barcodes. For each 500 qPCR reaction, $10^{-12} \mathrm{~g}(\sim 450,000$ copies $)$ of the pIDTSMART-AMP plasmid backbone 501 containing the Btk barcode indicated was used as DNA template. Threshold cycle $(\mathrm{Ct}) \mathrm{values}$ 502 shown are the median of three replicates; ND, Not determinable.

$503{ }^{a} 1 / 3$ replicates gave a $\mathrm{Ct}$ value of 37.087 


\section{References}

1. Yan R-C, P. WR. Trends and perspectives in animal $\square$ attached remote sensing. Frontiers in Ecology and the Environment. 2005;3(8):437-44.

2. Gibbons WJ, Andrews KM. PIT Tagging: Simple Technology at Its Best. BioScience. 2004;54(5):447-54.

3. Glandorf DC, van der Sluis I, Anderson AJ, Bakker PA, Schippers B. Agglutination, adherence, and root colonization by fluorescent pseudomonads. Appl Environ Microbiol. 1994;60(6):1726-33. 4. Glandorf DC, Verheggen P, Jansen T, Jorritsma JW, Smit E, Leeflang P, et al. Effect of genetically modified Pseudomonas putida WCS358r on the fungal rhizosphere microflora of field-grown wheat. Appl Environ Microbiol. 2001;67(8):3371-8.

5. De Leij F, Sutton EJ, Whipps JM, Fenlon JS, Lynch JM. Impact of Field Release of Genetically Modified Pseudomonas fluorescens on Indigenous Microbial Populations of Wheat. Appl Environ Microbiol. 1995;61(9):3443-53.

6. Krishnamurthy K, Gnanamanickam SS. Biological Control of Rice Blast by Pseudomonas fluorescens Strain Pf7-14: Evaluation of a Marker Gene and Formulations. Biological Control. 1998;13(3):158-65.

7. Moenne-Loccoz Y, Powell J, Higgins P, McCarthy J, O'Gara F. An investigation of the impact of biocontrol Pseudomonas fluorescens F113 on the growth of sugarbeet and the performance of subsequent clover-Rhizobium symbiosis. Appl Soil Ecol. 1998;7(3):225-37.

8. De Leij F, Thomas CE, Bailey MJ, Whipps JM, Lynch JM. Effect of Insertion Site and Metabolic Load on the Environmental Fitness of a Genetically Modified Pseudomonas fluorescens Isolate. Appl Environ Microbiol. 1998;64(7):2634-8.

9. Hensel M, Shea J, Gleeson C, Jones M, Dalton E, Holden D. Simultaneous identification of bacterial virulence genes by negative selection. Science. 1995;269(5222):400-3.

530 10. Mazurkiewicz P, Tang CM, Boone C, Holden DW. Signature-tagged mutagenesis: barcoding mutants for genome-wide screens. Nat Rev Genet. 2006;7(12):929-39.

11. Grant AJ, Restif O, McKinley TJ, Sheppard M, Maskell DJ, Mastroeni P. Modelling within-host spatiotemporal dynamics of invasive bacterial disease. PLoS Biol. 2008;6(4):e74.

534 12. Mastroeni P, Grant A. Dynamics of spread of Salmonella enterica in the systemic compartment. 535 Microbes Infect. 2013;15(13):849-57.

536 13. Varble A, Albrecht RA, Backes S, Crumiller M, Bouvier NM, Sachs D, et al. Influenza A virus 537 transmission bottlenecks are defined by infection route and recipient host. Cell host \& microbe.

538 2014;16(5):691-700.

539 14. Lauring AS, Andino R. Exploring the fitness landscape of an RNA virus by using a universal 540 barcode microarray. J Virol. 2011;85(8):3780-91.

541 15. Melton-Witt JA, McKay SL, Portnoy DA. Development of a Single-Gene, Signature-Tag-Based

542 Approach in Combination with Alanine Mutagenesis To Identify Listeriolysin O Residues Critical for the

543 In Vivo Survival of Listeria monocytogenes. Infection and Immunity. 2012;80(6):2221-30.

544 16. Melton-Witt JA, Rafelski SM, Portnoy DA, Bakardjiev AI. Oral infection with signature-tagged 545 Listeria monocytogenes reveals organ-specific growth and dissemination routes in guinea pigs. Infect

546 Immun. 2012;80(2):720-32.

547 17. Rego RO, Bestor A, Stefka J, Rosa PA. Population bottlenecks during the infectious cycle of the 548 Lyme disease spirochete Borrelia burgdorferi. PLoS One. 2014;9(6):e101009.

549 18. Gerlini A, Colomba L, Furi L, Braccini T, Manso AS, Pammolli A, et al. The role of host and 550 microbial factors in the pathogenesis of pneumococcal bacteraemia arising from a single bacterial cell 551 bottleneck. PLoS Pathog. 2014;10(3):e1004026. 
19. Martin CJ, Cadena AM, Leung VW, Lin PL, Maiello P, Hicks N, et al. Digitally Barcoding Mycobacterium tuberculosis Reveals In Vivo Infection Dynamics in the Macaque Model of Tuberculosis. MBio. 2017;8(3).

20. Abel S, Abel zur Wiesch P, Davis BM, Waldor MK. Analysis of Bottlenecks in Experimental Models of Infection. PLoS Pathog. 2015;11(6):e1004823.

21. Leitenberg M, Zilinskas RA. The Soviet Biological Weapons Program: A History. Cambridge, MA: Harvard University Press; 2012. 960 p.

22. Greenberg DL, Busch JD, Keim P, Wagner DM. Identifying experimental surrogates for Bacillus anthracis spores: a review. Investig Genet. 2010;1(1):4.

23. Emanuel PA, Buckley PE, Sutton TA, Edmonds JM, Bailey AM, Rivers BA, et al. Detection and tracking of a novel genetically tagged biological simulant in the environment. Appl Environ Microbiol. 2012;78(23):8281-8.

24. Buckley P, Rivers B, Katoski S, Kim MH, Kragl FJ, Broomall S, et al. Genetic barcodes for improved environmental tracking of an anthrax simulant. Appl Environ Microbiol. 2012;78(23):8272-80.

25. Bishop AH, Stapleton HL. Aerosol and Surface Deposition Characteristics of Two Surrogates for Bacillus anthracis Spores. Appl Environ Microbiol. 2016;82(22):6682-90.

568 26. Bishop AH, Robinson CV. Bacillus thuringiensis HD-1 Cry- : development of a safe, non-

569 insecticidal simulant for Bacillus anthracis. J Appl Microbiol. 2014;117(3):654-62.

$57027 . \quad$ Merrill L, Dunbar J, Richardson J, Kuske CR. Composition of bacillus species in aerosols from

57111 U.S. cities. J Forensic Sci. 2006;51(3):559-65.

572 28. Van Cuyk S, Duval N, Omberg KM. Bacillus thuringiensis: Presence and Persistence in the

573 Environment. Los Alamos National Laboratory, 2008 LA-UR-08-04878. biopesticide. Journal of Applied Microbiology. 2006;101(3):616-9.

30. Van Cuyk S, Deshpande A, Hollander A, Duval N, Ticknor L, Layshock J, et al. Persistence of Bacillus thuringiensis subsp. kurstaki in Urban Environments following Spraying. Applied and Environmental Microbiology. 2011;77(22):7954-61.

31. Sahl JW, Vazquez AJ, Hall CM, Busch JD, Tuanyok A, Mayo M, et al. The Effects of Signal Erosion and Core Genome Reduction on the Identification of Diagnostic Markers. mBio. 2016;7(5):e00846-16.

32. Gardner SN, Frey KG, Redden CL, Thissen JB, Allen JE, Allred AF, et al. Targeted amplification for enhanced detection of biothreat agents by next-generation sequencing. BMC research notes. 2015;8:682-.

33. Johnson SL, Daligault HE, Davenport KW, Jaissle J, Frey KG, Ladner JT, et al. Complete genome sequences for 35 biothreat assay-relevant bacillus species. Genome Announc. 2015;3(2).

34. Holden MT, Titball RW, Peacock SJ, Cerdeno-Tarraga AM, Atkins T, Crossman LC, et al. Genomic plasticity of the causative agent of melioidosis, Burkholderia pseudomallei. Proc Natl Acad Sci U S A. 2004;101(39):14240-5.

35. Parkhill J, Wren BW, Thomson NR, Titball RW, Holden MT, Prentice MB, et al. Genome sequence of Yersinia pestis, the causative agent of plague. Nature. 2001;413(6855):523-7.

36. Sebaihia M, Peck MW, Minton NP, Thomson NR, Holden MT, Mitchell WJ, et al. Genome sequence of a proteolytic (Group I) Clostridium botulinum strain Hall A and comparative analysis of the clostridial genomes. Genome Res. 2007;17(7):1082-92.

37. Kralik P, Ricchi M. A Basic Guide to Real Time PCR in Microbial Diagnostics: Definitions, Parameters, and Everything. Frontiers in Microbiology. 2017;8:108.

38. Liss M, Daubert D, Brunner K, Kliche K, Hammes U, Leiherer A, et al. Embedding Permanent Watermarks in Synthetic Genes. PLOS ONE. 2012;7(8):e42465.

39. Heider D, Barnekow A. DNA watermarks: a proof of concept. BMC Mol Biol. 2008;9:40.

40. Gibson DG, Benders GA, Andrews-Pfannkoch C, Denisova EA, Baden-Tillson H, Zaveri J, et al. Complete chemical synthesis, assembly, and cloning of a Mycoplasma genitalium genome. Science. 2008;319(5867):1215-20. 
603 41. Jupiter DC, Ficht TA, Samuel J, Qin QM, de Figueiredo P. DNA watermarking of infectious

604 agents: progress and prospects. PLoS Pathog. 2010;6(6):e1000950.

605 42. Torok TJ, Tauxe RV, Wise RP, Livengood JR, Sokolow R, Mauvais S, et al. A large community

606 outbreak of salmonellosis caused by intentional contamination of restaurant salad bars. JAMA.

607 1997;278(5):389-95.

608 43. Anonymous. Amerithrax Investigative Summary. Washington, DC: Department of Justice, 2010.

609 44. Rasko DA, Worsham PL, Abshire TG, Stanley ST, Bannan JD, Wilson MR, et al. Bacillus

610 anthracis comparative genome analysis in support of the Amerithrax investigation. Proc Natl Acad Sci U

611 S A. 2011;108(12):5027-32.

612 45. Plaut RD, Stibitz S. Improvements to a Markerless Allelic Exchange System for Bacillus

613 anthracis. PLoS One. 2015;10(12):e0142758.

614 46. Cote CK, Buhr T, Bernhards CB, Bohmke MD, Calm AM, Esteban-Trexler JS, et al. A Standard

615 Method To Inactivate Bacillus anthracis Spores to Sterility via Gamma Irradiation. Appl Environ

616 Microbiol. 2018;84(12).

617 47. Wattam AR, Abraham D, Dalay O, Disz TL, Driscoll T, Gabbard JL, et al. PATRIC, the bacterial

618 bioinformatics database and analysis resource. Nucleic Acids Res. 2014;42(Database issue):D581-91.

619 48. Sun W, Wang S, Curtiss R, 3rd. Highly efficient method for introducing successive multiple

620 scarless gene deletions and markerless gene insertions into the Yersinia pestis chromosome. Appl Environ

621 Microbiol. 2008;74(13):4241-5.

622 49. Datsenko KA, Wanner BL. One-step inactivation of chromosomal genes in Escherichia coli K-12

623 using PCR products. Proc Natl Acad Sci U S A. 2000;97(12):6640-5.

624 50. Horton RM, Hunt HD, Ho SN, Pullen JK, Pease LR. Engineering hybrid genes without the use of

625 restriction enzymes: gene splicing by overlap extension. Gene. 1989;77(1):61-8. 


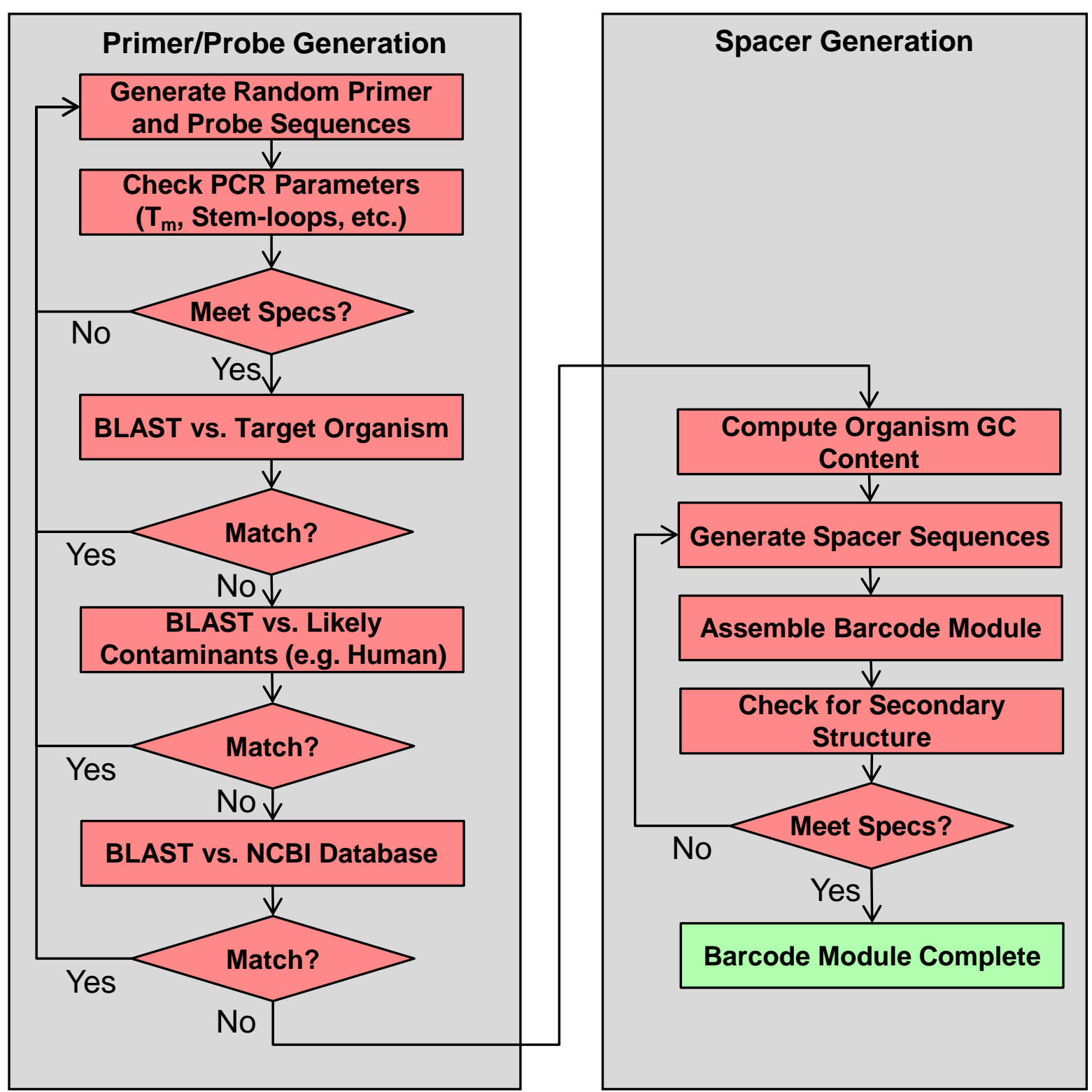



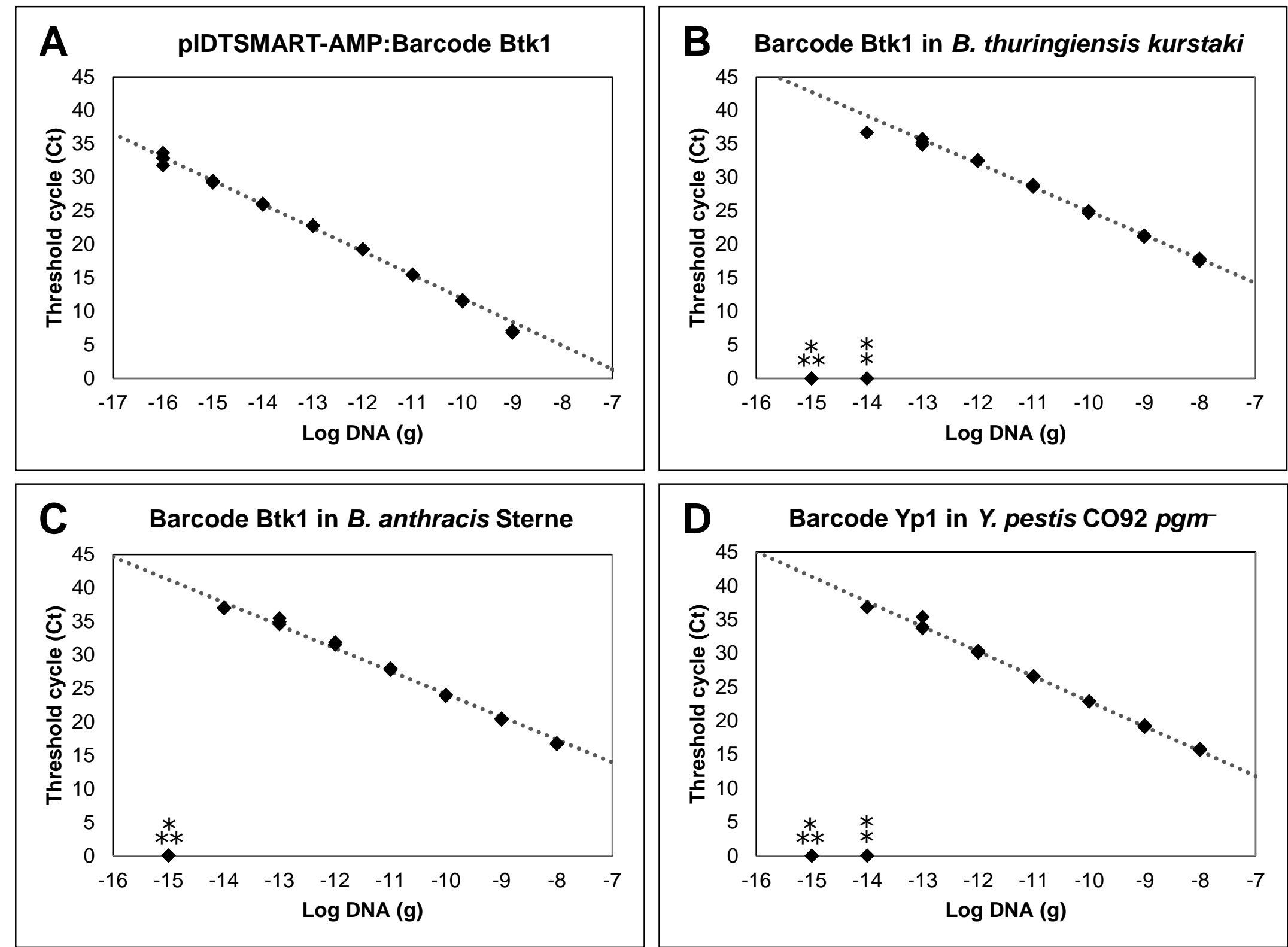

Figure 2 
DNA Template

\begin{tabular}{|c|c|c|c|c|c|c|c|c|c|c|c|c|}
\hline & 1 & 2 & 3 & 4 & 5 & 6 & 7 & 8 & 9 & 10 & 11 & 12 \\
\hline 1 & 23.0 & ND & ND & ND & ND & ND & ND & ND & ND & ND & ND & ND \\
\hline 2 & ND & 24.5 & ND & ND & ND & ND & ND & ND & ND & ND & ND & ND \\
\hline 3 & ND & ND & 22.5 & ND & ND & ND & ND & ND & ND & ND & ND & ND \\
\hline 4 & ND & ND & ND & 23.4 & ND & ND & ND & ND & ND & ND & ND & ND \\
\hline 5 & ND & ND & ND & ND & 24.5 & ND & ND & ND & ND & ND & ND & ND \\
\hline 6 & ND & ND & ND & ND & ND & 23.9 & ND & ND & ND & ND & ND & ND \\
\hline 7 & ND & ND & ND & ND & ND & ND & 24.0 & ND & ND & ND & ND & ND \\
\hline 8 & ND & ND & ND & ND & ND & ND & ND & 22.7 & ND & ND & ND & $\mathrm{ND}$ \\
\hline 9 & ND & ND & ND & ND & ND & ND & ND & ND & 20.4 & ND & ND & $\mathrm{ND}$ \\
\hline 10 & ND & ND & ND & ND & ND & ND & ND & ND & ND & 19.3 & $\mathrm{ND}^{a}$ & $\mathrm{ND}$ \\
\hline 11 & ND & ND & ND & ND & ND & ND & ND & ND & ND & ND & 18.7 & $\mathrm{ND}$ \\
\hline 12 & ND & ND & ND & ND & ND & ND & ND & ND & ND & ND & ND & 19.6 \\
\hline
\end{tabular}

